\title{
Asymptotic behavior of second-order nonlinear neutral dynamic equations
}

\author{
Yefei Jing, Chenghui Zhang* and Tongxing Li
}

\section{*Correspondence:}

zchui@sdu.edu.cn

School of Control Science and

Engineering, Shandong University,

Jinan, Shandong 250061, P.R. China

\begin{abstract}
This paper is concerned with oscillation and asymptotic behavior of a second-order neutral delay dynamic equation on an arbitrary time scale. We obtain two theorems which guarantee that every solution of the studied equation oscillates or converges to zero. These results improve and complement some known results given in the literature.

MSC: 34K11;34N05; 39A10; 39A12; 39A13; 39A21

Keywords: asymptotic behavior; oscillation; neutral delay dynamic equation; time scale
\end{abstract}

\section{Introduction}

In this paper, we study oscillation and asymptotic behavior of a second-order nonlinear neutral delay dynamic equation

$$
\left(r(t)\left((x(t)+p(t) x(\eta(t)))^{\Delta}\right)^{\gamma}\right)^{\Delta}+f(t, x(g(t)))=0
$$

on an arbitrary time scale $\mathbb{T}$, where $\gamma$ is a quotient of odd positive integers, $r$ and $p$ are positive rd-continuous functions on $\mathbb{T}, 0 \leq p(t) \leq p_{1}<1$. Also, we assume that $\eta, g: \mathbb{T} \rightarrow \mathbb{T}$ are rd-continuous, $\eta(t) \leq t, g(t) \leq t, \lim _{t \rightarrow \infty} \eta(t)=\lim _{t \rightarrow \infty} g(t)=\infty, u f(t, u)>0$ for all $u \neq 0$, and there exists a positive rd-continuous function $q$ defined on $\mathbb{T}$ such that $|f(t, u)| \geq$ $q(t)|u|^{\gamma}$.

The theory of dynamic equations on time scales, which goes back to its founder Hilger [1], has recently attracted attention of researchers. Several authors have expounded on various aspects of this new theory; see the survey paper written by Agarwal et al. [2] and the references cited therein. The books on the subject of time scales, by Bohner and Peterson $[3,4]$, present much of time scale calculus.

Since we are interested in oscillatory and asymptotic properties, we assume throughout this paper that the given time scale $\mathbb{T}$ is unbounded above. We assume that $t_{0} \in \mathbb{T}$, and it is convenient to assume that $t_{0}>0$, and define the time scale interval of the form $\left[t_{0}, \infty\right)_{\mathbb{T}}$ by $\left[t_{0}, \infty\right)_{\mathbb{T}}:=\left[t_{0}, \infty\right) \cap \mathbb{T}$. Throughout, we use the notation $z:=x+p x \circ \eta$. By a solution of equation (1.1), we mean a non-trivial real-valued function $x \in \mathrm{C}_{\mathrm{rd}}^{1}\left[T_{x}, \infty\right)_{\mathbb{T}}, T_{x} \in\left[t_{0}, \infty\right)_{\mathbb{T}}$ which has the property that $z$ and $r\left(z^{\Delta}\right)^{\gamma}$ are defined and $\Delta$-differentiable for $t \in \mathbb{T}$ and satisfies equation $(1.1)$ on $\left[T_{x}, \infty\right)_{\mathbb{T}}$. The solutions vanishing in some neighborhood of infinity will be excluded from our consideration. A solution $x$ of equation (1.1) is said to be

\section{Springer}

○2013 Jing et al.; licensee Springer. This is an Open Access article distributed under the terms of the Creative Commons Attribution License (http://creativecommons.org/licenses/by/2.0), which permits unrestricted use, distribution, and reproduction in any medium, provided the original work is properly cited. 
oscillatory if it is neither eventually positive nor eventually negative; otherwise, it is called nonoscillatory.

In recent years, there has been much research activity concerning oscillation and nonoscillation of solutions to neutral differential and dynamic equations on time scales, we refer the reader to [5, 6] and [7-22], and the references cited therein. Han et al. [6] studied a second-order nonlinear neutral equation

$$
\left(r(t)\left|u^{\prime}(t)\right|^{\alpha-1} u^{\prime}(t)\right)^{\prime}+q(t) f(x(\delta(t)))=0,
$$

where $u:=x+p x \circ \tau$, and established two results which guarantee that every solution of equation (1.2) is oscillatory under the assumptions that

$$
p^{\prime}(t) \geq 0, \quad \sigma(t) \leq \tau(t)=t-\tau_{0} \leq t,
$$

and

$$
\int_{t_{0}}^{\infty} \frac{\mathrm{d} t}{r^{1 / \gamma}(t)}<\infty
$$

Agarwal et al. [7], Erbe et al. [8], Şahiner [9], Saker [10], Saker et al. [11], Saker and O’Regan [12], Chen [13], Zhang and Wang [14], Wu et al. [15], Candan [17], and Li et al. [19] investigated equation (1.1) and obtained some oscillation criteria in the case

$$
\int_{t_{0}}^{\infty} \frac{\Delta t}{r^{1 / \gamma}(t)}=\infty
$$

As yet, there are few results regarding the study of asymptotic behavior of equation (1.1) under the assumption that

$$
\int_{t_{0}}^{\infty} \frac{\Delta t}{r^{1 / \gamma}(t)}<\infty
$$

In 2007, Saker et al. [11] posed an open problem as follows: How to establish oscillation criteria for equation (1.1) when condition (1.5) holds? Assuming (1.5), Zhang et al. [21, 22] obtained some sufficient conditions which insure that all solutions of equation (1.1) are oscillatory.

The purpose of this paper is to present some asymptotic tests for equation (1.1) in the case where (1.5) holds. This paper is organized as follows: In the next section, we shall establish the main results. In Section 3, two examples are provided to illustrate the results obtained.

In the sequel, when we write a functional inequality without specifying its domain of validity, we assume that it holds for all sufficiently large $t$.

\section{Main results}

In what follows, we use the notation

$$
\delta_{+}^{\Delta}(t):=\max \left\{0, \delta^{\Delta}(t)\right\}, \quad Q(t):=q(t)(1-p(g(t)))^{\gamma}, \quad \theta(t, u):=\frac{\int_{u}^{g(t)} \Delta s / r^{1 / \gamma}(s)}{\int_{u}^{t} \Delta s / r^{1 / \gamma}(s)},
$$

and, for sufficiently large $T_{*}, \beta\left(t, T_{*}\right):=\theta^{\gamma}\left(t, T_{*}\right)$. 
In order to prove our main results, we will use the following result; see [8, Theorem 2.1].

Theorem 2.1 Let (1.4) hold. Suppose that there exists a positive $\Delta$-differentiable function $\delta$ such that for all sufficiently large $T *$ and for $g(T)>T *$,

$$
\limsup _{t \rightarrow \infty} \int_{T}^{t}\left(\beta\left(s, T_{*}\right) \delta(s) Q(s)-\frac{r(s)\left(\delta_{+}^{\Delta}(s)\right)^{\gamma+1}}{(\gamma+1)^{\gamma+1} \delta^{\gamma}(s)}\right) \Delta s=\infty
$$

Then every solution $x$ of equation (1.1) is oscillatory.

Theorem 2.2 Let (1.5) hold. Assume that there exists a positive $\Delta$-differentiable function $\delta$ such that for all sufficiently large $T *$ and for $g(T)>T *$, one has (2.1). If

$$
\int_{t_{0}}^{\infty} \frac{1}{r^{1 / \gamma}(s)}\left(\int_{t_{0}}^{s} q(u)\left(\int_{g(u)}^{\infty} \frac{\Delta v}{r^{1 / \gamma}(v)}\right)^{\gamma} \Delta u\right)^{1 / \gamma} \Delta s=\infty
$$

then every solution $x$ of equation (1.1) is oscillatory or $\lim _{t \rightarrow \infty} x(t)=0$.

Proof Let $x$ be a nonoscillatory solution of equation (1.1). Without loss of generality, we assume that $x(t)>0, x(\eta(t))>0$, and $x(g(t))>0$ for $t \in\left[t_{0}, \infty\right)_{\mathbb{T}}$. Then $z(t)>0$ for $t \in$ $\left[t_{0}, \infty\right)_{\mathbb{T}}$. In view of $(1.1)$, we get

$$
\left(r(t)\left(z^{\Delta}(t)\right)^{\gamma}\right)^{\Delta} \leq-q(t) x^{\gamma}(g(t))<0, \quad t \in\left[t_{0}, \infty\right)_{\mathbb{T}} .
$$

Therefore, $r\left(z^{\Delta}\right)^{\gamma}$ is strictly decreasing, and there exists a $t_{1} \in\left[t_{0}, \infty\right)_{\mathbb{T}}$ such that $z^{\Delta}(t)>0$ or $z^{\Delta}(t)<0$ for $t \in\left[t_{1}, \infty\right)_{\mathbb{T}}$. We consider each of two cases separately.

Case 1. Assume that $z^{\Delta}(t)>0$ for $t \in\left[t_{1}, \infty\right)_{\mathbb{T}}$. As in the proof of [8, Theorem 2.1], we can obtain a contradiction to (2.1).

Case 2. Assume that $z^{\Delta}(t)<0$ for $t \in\left[t_{1}, \infty\right)_{\mathbb{T}}$. Then, there exists a finite limit

$$
\lim _{t \rightarrow \infty} z(t)=l
$$

where $l \geq 0$. Now, we claim that $l=0$. If not, then for any $\epsilon>0$, we have $l<z(t)<l+\epsilon$, eventually. Take $0<\epsilon<l\left(1-p_{1}\right) / p_{1}$. We calculate

$$
x(t)=z(t)-p(t) x(\eta(t))>l-p_{1} z(\eta(t))>l-p_{1}(l+\epsilon)=m(l+\epsilon)>m z(t),
$$

where

$$
m:=\frac{l}{l+\epsilon}-p_{1}=\frac{l\left(1-p_{1}\right)-\epsilon p_{1}}{l+\epsilon}>0 .
$$

Since $r\left(z^{\Delta}\right)^{\gamma}$ is strictly decreasing,

$$
z^{\Delta}(s) \leq \frac{r^{1 / \gamma}(t) z^{\Delta}(t)}{r^{1 / \gamma}(s)}, \quad s \in[t, \infty)_{\mathbb{T}} .
$$

Integrating the inequality above from $t$ to $l$ and letting $l \rightarrow \infty$, we have by (2.3) that

$$
z(t) \geq-r^{1 / \gamma}(t) z^{\Delta}(t) \int_{t}^{\infty} \frac{\Delta s}{r^{1 / \gamma}(s)} \geq-r^{1 / \gamma}\left(t_{1}\right) z^{\Delta}\left(t_{1}\right) \int_{t}^{\infty} \frac{\Delta s}{r^{1 / \gamma}(s)}=k \int_{t}^{\infty} \frac{\Delta s}{r^{1 / \gamma}(s)},
$$


where $k:=-r^{1 / \gamma}\left(t_{1}\right) z^{\Delta}\left(t_{1}\right)>0$. Combining (2.4) and (2.5), we get

$$
x(g(t)) \geq m z(g(t)) \geq m k \int_{g(t)}^{\infty} \frac{\Delta s}{r^{1 / \gamma}(s)} .
$$

Then by (2.3), we obtain

$$
\left(r(t)\left(-z^{\Delta}(t)\right)^{\gamma}\right)^{\Delta} \geq(m k)^{\gamma} q(t)\left(\int_{g(t)}^{\infty} \frac{\Delta s}{r^{1 / \gamma}(s)}\right)^{\gamma} .
$$

Integrating the inequality above from $t_{2}\left(t_{2} \in\left[t_{1}, \infty\right)_{\mathbb{T}}\right)$ to $t$, we have

$$
\begin{aligned}
r(t)\left(-z^{\Delta}(t)\right)^{\gamma} & \geq r\left(t_{2}\right)\left(-z^{\Delta}\left(t_{2}\right)\right)^{\gamma}+(m k)^{\gamma} \int_{t_{2}}^{t} q(s)\left(\int_{g(s)}^{\infty} \frac{\Delta u}{r^{1 / \gamma}(u)}\right)^{\gamma} \Delta s \\
& \geq(m k)^{\gamma} \int_{t_{2}}^{t} q(s)\left(\int_{g(s)}^{\infty} \frac{\Delta u}{r^{1 / \gamma}(u)}\right)^{\gamma} \Delta s,
\end{aligned}
$$

which implies that

$$
z^{\Delta}(t) \leq-\frac{m k}{r^{1 / \gamma}(t)}\left(\int_{t_{2}}^{t} q(s)\left(\int_{g(s)}^{\infty} \frac{\Delta u}{r^{1 / \gamma}(u)}\right)^{\gamma} \Delta s\right)^{1 / \gamma} .
$$

Integrating the latter inequality from $t_{2}$ to $t$, we get

$$
z(t) \leq z\left(t_{2}\right)-m k \int_{t_{2}}^{t} \frac{1}{r^{1 / \gamma}(s)}\left(\int_{t_{2}}^{s} q(u)\left(\int_{g(u)}^{\infty} \frac{\Delta v}{r^{1 / \gamma}(v)}\right)^{\gamma} \Delta u\right)^{1 / \gamma} \Delta s,
$$

which yields $\lim _{t \rightarrow \infty} z(t)=-\infty$, this is a contradiction. Hence, $\lim _{t \rightarrow \infty} z(t)=0$. By virtue of $0<x(t) \leq z(t), \lim _{t \rightarrow \infty} x(t)=0$. The proof is complete.

Next, we establish another criterion which improves Theorem 2.2.

Theorem 2.3 Let (1.5) hold. Suppose that there exists a positive $\Delta$-differentiable function $\delta$ such that for all sufficiently large $T *$ and for $g(T)>T *$, one has (2.1). If

$$
\int_{t_{0}}^{\infty}\left(\frac{1}{r(s)} \int_{t_{0}}^{s} q(u) \Delta u\right)^{1 / \gamma} \Delta s=\infty
$$

then every solution $x$ of equation (1.1) is oscillatory or $\lim _{t \rightarrow \infty} x(t)=0$.

Proof Let $x$ be a nonoscillatory solution of equation (1.1). Without loss of generality, we assume that $x(t)>0, x(\eta(t))>0$, and $x(g(t))>0$ for $t \in\left[t_{0}, \infty\right)_{\mathbb{T}}$. Then $z(t)>0$ for $t \in$ $\left[t_{0}, \infty\right)_{\mathbb{T}}$. In view of (1.1), we get (2.3). Thus, $r\left(z^{\Delta}\right)^{\gamma}$ is strictly decreasing, and there exists a $t_{1} \in\left[t_{0}, \infty\right)_{\mathbb{T}}$ such that $z^{\Delta}(t)>0$ or $z^{\Delta}(t)<0$ for $t \in\left[t_{1}, \infty\right)_{\mathbb{T}}$. We consider each of two cases separately.

Case 1. Assume that $z^{\Delta}(t)>0$ for $t \in\left[t_{1}, \infty\right)_{\mathbb{T}}$. Similarly to the proof of [8, Theorem 2.1], we can obtain a contradiction to (2.1).

Case 2. Assume that $z^{\Delta}(t)<0$ for $t \in\left[t_{1}, \infty\right)_{\mathbb{T}}$. Then there exists a finite limit

$$
\lim _{t \rightarrow \infty} z(t)=l,
$$


where $l \geq 0$. Next, we claim that $l=0$. If not, then for any $\epsilon>0$, we have $l<z(t)<l+\epsilon$, eventually. Take $0<\epsilon<l\left(1-p_{1}\right) / p_{1}$. Then we have (2.4). It follows from (2.3), (2.4), and $z(g(t))>l$ that

$$
\left(r(t)\left(z^{\Delta}(t)\right)^{\gamma}\right)^{\Delta} \leq-q(t) x^{\gamma}(g(t)) \leq-m^{\gamma} q(t) z^{\gamma}(g(t)) \leq-(m l)^{\gamma} q(t) .
$$

Integrating the inequality above from $t_{2}\left(t_{2} \in\left[t_{1}, \infty\right)_{\mathbb{T}}\right)$ to $t$, we get

$$
r(t)\left(z^{\Delta}(t)\right)^{\gamma}-r\left(t_{2}\right)\left(z^{\Delta}\left(t_{2}\right)\right)^{\gamma} \leq-(m l)^{\gamma} \int_{t_{2}}^{t} q(s) \Delta s
$$

which yields

$$
z^{\Delta}(t) \leq-m l\left(\frac{1}{r(t)} \int_{t_{2}}^{t} q(s) \Delta s\right)^{1 / \gamma}
$$

Integrating the latter inequality from $t_{2}$ to $t$, we have

$$
z(t) \leq z\left(t_{2}\right)-m l \int_{t_{2}}^{t}\left(\frac{1}{r(s)} \int_{t_{2}}^{s} q(u) \Delta u\right)^{1 / \gamma} \Delta s
$$

which implies that $\lim _{t \rightarrow \infty} z(t)=-\infty$, this is a contradiction. Hence, $\lim _{t \rightarrow \infty} z(t)=0$. By $0<x(t) \leq z(t), \lim _{t \rightarrow \infty} x(t)=0$. This completes the proof.

Remark 2.1 When $\mathbb{T}=\mathbb{R}$, Theorems 2.2 and 2.3 improve results of Han et al. [6, Theorems 2.1 and 2.2] since our results do not require condition (1.3).

Remark 2.2 The results obtained in this paper complement the recent results given in [7-19] in the sense that these results can be applied to case (1.5).

\section{Applications}

In this section, we give two examples to illustrate applications of results in the previous section.

Example 3.1 For $t \in[1, \infty)_{\mathbb{T}}$, consider a second-order neutral delay dynamic equation

$$
\left(\frac{1}{t \sigma(t)}\left(x(t)+\frac{1}{2} x(\eta(t))\right)^{\Delta}\right)^{\Delta}+q(t) x(g(t))=0
$$

where $q(t) \geq \beta>0$ satisfying $\int_{1}^{t}(q(u) / g(u)) \Delta u \geq \sigma(t), \eta(t) \leq t, g(t) \leq t$, and $\lim _{t \rightarrow \infty} \eta(t)=$ $\lim _{t \rightarrow \infty} g(t)=\infty$. Let $\gamma=1$ and $r(t)=1 /(t \sigma(t))$. Then, we have

$$
\int_{t_{0}}^{\infty} \frac{\Delta t}{r^{1 / \gamma}(t)}=\int_{1}^{\infty} \frac{\Delta t}{t \sigma(t)}=1,
$$

that is, (1.5) holds. Note that $Q(t)=q(t) / 2$, and for every constant $k \in(0,1)$ and for $t \in$ $\left[t_{k}, \infty\right)_{\mathbb{T}}$,

$$
\beta\left(t, T_{*}\right)=\left(\frac{\frac{1}{T_{*}}-\frac{1}{g(t)}}{\frac{1}{T_{*}}-\frac{1}{t}}\right)^{\gamma}=\frac{t}{g(t)} \frac{g(t)-T_{*}}{t-T_{*}} \geq k>0 .
$$


Choose $\delta(t)=1$. It is not difficult to verify that (2.1) holds. On the other hand,

$$
\int_{t_{0}}^{\infty} \frac{1}{r^{1 / \gamma}(s)}\left(\int_{t_{0}}^{s} q(u)\left(\int_{g(u)}^{\infty} \frac{\Delta v}{r^{1 / \gamma}(v)}\right)^{\gamma} \Delta u\right)^{1 / \gamma} \Delta s \geq \int_{1}^{\infty} \frac{\Delta s}{s}=\infty
$$

Thus, we have by Theorem 2.2 that every solution $x$ of (3.1) is oscillatory or $\lim _{t \rightarrow \infty} x(t)=$ 0 .

Example 3.2 For $t \in[1, \infty)_{\mathbb{T}}$, consider a second-order neutral delay dynamic equation

$$
\left(\frac{1}{(t \sigma(t))^{\gamma}}\left(\left(x(t)+\frac{1}{2} x(\eta(t))\right)^{\Delta}\right)^{\gamma}\right)^{\Delta}+q(t) x^{\gamma}(g(t))=0,
$$

where $q(t) \geq \beta>0$ satisfying $\int_{1}^{t} q(u) \Delta u \geq \sigma^{\gamma}(t), \eta(t) \leq t, g(t) \leq t$, and $\lim _{t \rightarrow \infty} \eta(t)=$ $\lim _{t \rightarrow \infty} g(t)=\infty$. Let $r(t)=1 /(t \sigma(t))^{\gamma}$. Then we have

$$
\int_{t_{0}}^{\infty} \frac{\Delta t}{r^{1 / \gamma}(t)}=\int_{1}^{\infty} \frac{\Delta t}{t \sigma(t)}=1
$$

that is, (1.5) holds. Note that $Q(t)=q(t) / 2^{\gamma}$, and for every constant $k \in(0,1)$ and for $t \in$ $\left[t_{k}, \infty\right)_{\mathbb{T}}$,

$$
\beta\left(t, T_{*}\right)=\left(\frac{\frac{1}{T_{*}}-\frac{1}{g(t)}}{\frac{1}{T_{*}}-\frac{1}{t}}\right)^{\gamma}=\left(\frac{t}{g(t)} \frac{g(t)-T_{*}}{t-T_{*}}\right)^{\gamma} \geq k>0 .
$$

Choose $\delta(t)=1$. It is easy to verify that (2.1) holds. On the other hand,

$$
\int_{t_{0}}^{\infty}\left(\frac{1}{r(s)} \int_{t_{0}}^{s} q(u) \Delta u\right)^{1 / \gamma} \Delta s \geq \int_{1}^{\infty} \frac{\Delta s}{s}=\infty
$$

Hence, by Theorem 2.3, every solution $x$ of (3.2) is oscillatory or $\lim _{t \rightarrow \infty} x(t)=0$.

\section{Competing interests}

The authors declare that they have no competing interests.

Authors' contributions

All authors contributed equally to the writing of the paper. All authors read and approved the final manuscript.

\section{Acknowledgements}

This research is supported by the National Key Basic Research Program of P.R. China (2013CB035604) and the NNSF of P.R. China (Grant Nos. 61034007, 51277116, and 51107069).

Received: 31 July 2013 Accepted: 10 September 2013 Published: 08 Nov 2013

\section{References}

1. Hilger, S: Analysis on measure chains-a unified approach to continuous and discrete calculus. Results Math. 18, 18-56 (1990)

2. Agarwal, RP, Bohner, M, O'Regan, D, Peterson, A: Dynamic equations on time scales: a survey. J. Comput. Appl. Math. 141, 1-26 (2002)

3. Bohner, M, Peterson, A: Dynamic Equations on Time Scales: An Introduction with Applications. Birkhäuser, Boston (2001)

4. Bohner, M, Peterson, A: Advances in Dynamic Equations on Time Scales. Birkhäuser, Boston (2003)

5. Baculíková, B, Džurina, J: Oscillation of third-order neutral differential equations. Math. Comput. Model. 52, 215-226 (2010) 
6. Han, Z, Li, T, Sun, S, Sun, Y: Remarks on the paper [Appl. Math. Comput. 207 (2009) 388-396]. Appl. Math. Comput. 215, 3998-4007 (2010)

7. Agarwal, RP, O'Regan, D, Saker, SH: Oscillation criteria for second-order nonlinear neutral delay dynamic equations. J. Math. Anal. Appl. 300, 203-217 (2004)

8. Erbe, L, Hassan, TS, Peterson, A: Oscillation criteria for nonlinear functional neutral dynamic equations on time scales. J. Differ. Equ. Appl. 15, 1097-1116 (2009)

9. Şahiner, Y: Oscillation of second order neutral delay and mixed type dynamic equations on time scales. Adv. Differ. Equ. 2006, 1-9 (2006)

10. Saker, $\mathrm{SH}$ : Oscillation of second-order nonlinear neutral delay dynamic equations on time scales. J. Comput. Appl. Math. 187, 123-141 (2006)

11. Saker, SH, Agarwal, RP, O'Regan, D: Oscillation results for second-order nonlinear neutral delay dynamic equations on time scales. Appl. Anal. 86, 1-17 (2007)

12. Saker, $\mathrm{SH}, \mathrm{O}$ 'Regan, D: New oscillation criteria for second-order neutral functional dynamic equations via the generalized Riccati substitution. Commun. Nonlinear Sci. Numer. Simul. 16, 423-434 (2011)

13. Chen, DX: Oscillation of second-order Emden-Fowler neutral delay dynamic equations on time scales. Math. Comput. Model. 51, 1221-1229 (2010)

14. Zhang, SY, Wang, QR: Oscillation of second-order nonlinear neutral dynamic equations on time scales. Appl. Math. Comput. 216, 2837-2848 (2010)

15. Wu, HW, Zhuang, RK, Mathsen, RM: Oscillation criteria for second-order nonlinear neutral variable delay dynamic equations. Appl. Math. Comput. 178, 321-331 (2006)

16. Li, T, Han, Z, Sun, S, Yang, D: Existence of nonoscillatory solutions to second-order neutral delay dynamic equations on time scales. Adv. Differ. Equ. 2009, 1-10 (2009)

17. Candan, T: Oscillation criteria for second-order nonlinear neutral dynamic equations with distributed deviating arguments on time scales. Adv. Differ. Equ. 2013, 1-8 (2013)

18. Karpuz, B: Necessary and sufficient conditions on the asymptotic behavior of second-order neutral delay dynamic equations with positive and negative coefficients. Math. Methods Appl. Sci. (2013). doi:10.1002/mma.2884

19. Li, T, Agarwal, RP, Bohner, M: Some oscillation results for second-order neutral dynamic equations. Hacet. J. Math. Stat. 41, 715-721 (2012)

20. Yang, J: Oscillation criteria for certain third-order variable delay functional dynamic equations on time scales. J. Appl. Math. Comput. 43, 445-466 (2013)

21. Zhang, C, Agarwal, RP, Bohner, M, Li, T: New oscillation results for second-order neutral delay dynamic equations. Adv. Differ. Equ. 2012, 1-14 (2012)

22. Zhang, C, Agarwal, RP, Bohner, M, Li, T: Oscillation of second-order nonlinear neutral dynamic equations with noncanonical operators. Bull. Malays. Math. Sci. Soc. (2013, in press)

10.1186/1029-242X-2013-505

Cite this article as: Jing et al.: Asymptotic behavior of second-order nonlinear neutral dynamic equations. Journal of Inequalities and Applications 2013, 2013:505

\section{Submit your manuscript to a SpringerOpen ${ }^{\circ}$ journal and benefit from:}

- Convenient online submission

- Rigorous peer review

- Immediate publication on acceptance

- Open access: articles freely available online

- High visibility within the field

- Retaining the copyright to your article 